\title{
Ontological Model to Predict user Mobility
}

\author{
Atef Zaguia ${ }^{1}$, Roobaea Alroobaea ${ }^{2}$ \\ Computer Science, College of Computing and Information Technology, \\ Taif University \\ Taif, Saudi Arabia
}

\begin{abstract}
With the remarkable technological evolution of mobile devices, the use of computing resources has become possible at any time and independent of the geographical position of the user. This phenomenon has various names such as omnipresent diffuse computing, pervasive computing, or ubiquitous systems. This new form of computing allows users to have access to shared and ubiquitous services focused on their needs, and it is based on context prediction, especially the prediction of the user's location. This paper aims to present a new approach for predicting a user's next probable location, by presenting an ontological model which is based on the pattern technique. This is carried out by using an ontological model that comprises different user behaviors and presents details about the environment, where the user is located. The results after tested on real data show that the presented ontological model was able to achieve $85 \%$ future location-prediction accuracy (in the case of no similar patterns). Future work will focus on the integration of the Bayesian network to improve the results. This approach will be implemented in smart homes or smart cities to reduce energy consumption.
\end{abstract}

Keywords-Context prediction; pervasive system; context-aware system; pattern; ontology; ontological model

\section{INTRODUCTION}

Advances in technology have recently led to the emergence of more complicated computing systems. Small devices such as smartphones and other devices providing personal digital assistance have become part and parcel of people's daily life. Such devices come to facilitate user's access to information anywhere, anytime. Regardless of their geographical location, users can exchange data easier and quickly. These qualities made the newly invented devices omnipresent. The fact led to the emergence of a new trend of systems, namely, ubiquitous computing or pervasive systems.

The term "ubiquitous computing" was introduced by Mark Weiser, who defined it as many computers serving each person [1]. His vision about ubiquitous computing is summed up as follows: "In the twenty-first century the technology revolution will move into the everyday, the small and the invisible"[1]. He emphasized that the effect of technology will be grew tenfold because they will become a part of daily life. Also. he said that "as technology becomes more imbedded and invisible, it calms our lives by removing annoyances while keeping us connected with what is truly important" [1]. Furthermore, he mentions that in the 21st century, the computers will act as an active and smarter companion rather than an ordinary office assistant. They will go beyond the form of an omnipresent physical infrastructure to an intelligent ambient infrastructure that assists its users in an active and intelligent manner.
In daily life, a variety of pervasive systems, as predicted by Mark Weiser, are already used: smart glasses [2], interactive maps [3], wearable computers [4], magnifying glass [5], mobile healthcare industry [6] etc.

To offer better services to users, these systems should be aware of contexts (context awareness) [7], so that a system can adapt automatically to a context [8], [9], [10]. Many researches have focused on this field, especially on context prediction, and more specific, location prediction. Depending on prediction, the system will be capable to prepare adequate services to be offered to the user.

The rest of this paper is organized as follows: Section II discusses work related to context prediction. Section III presents the various components of the proposed architecture. Section IV describes the approach to predict future location. Section V shows the use case and results. The conclusion is presented in Section VI.

\section{RELATED WORK}

According to the widely acknowledged definition, context is "any information that can be used to characterize a situation of an entity. An entity is a person, place, or object that is considered relevant to the interaction between a user and an application, including the user and an application and application themselves" [11]. In other words, context is any piece of information used to describe the situation of an entity that can be a place, a person, or an object. The aspects of context comprise, but are not restricted to, location, weather, identity, activity, and time [12].

Context prediction is one of the most imperative tasks in the field of ubiquitous computing. The studies concerning model context or context [13], [9, 14] sensitivity have shown that predicting future features (context) has a direct influence on providing the most adequate services to the user without his/her direct involvement. Therefore, the system will be capable to proceed autonomously on behalf of users.

Several researches have focused on the future location of the user, such as [15], [16], [17], because it is considered an important contextual information. Its determination helps to provide the most appropriate services to the user: for example, a teacher in his/her office likes to find, for the next lecture (next location: class), that the computer and projector are turned on and that the slides are projected for the desired chapter.

The related works presented many techniques to predict future contexts, such as expert systems and decision trees [18], [19], [20]. These techniques are founded on rule-based engines and expert system and they aim to define the rules for 
prediction. They provide a very clear view of the system. They are simple to comprehend, and they can handle the non-linear interactions between contexts. They are not influenced by outliers and they can handle large categorical and numeric data. Markov chains [20], [21] is another technique. It is based on the decomposition of the context into a finite set of non-overlapping states. Using this method will determine a user's behaviors from the sequences of his/her actions record. The goal of this method is to determine the transition probability from one state to another using the following equation:

$P_{i j}=P\left(S(t+1)=S_{j} \mid S(t)=S i\right)$

Where: $i, j \in[0 . . n-1]$

$\mathrm{t}=0,1,2,3, \ldots$

Furthermore, the neural networks approach is one of the popular approaches for machine learning. It is inspired by the way natural nervous systems works, such as the brain [22, 23]. Many studies have used this approach to predict the next feature or context, such as Mikkluscak [24], Mozer [25], Vintan [26], Lin and al. [27], etc. Mozer [25] uses feedforward neural networks trained with back propagation to predict the most probable zone soon to be entered. This approach helps to estimate hot water usage. In [26], the authors also use the neural network to predict the next room number. "They chose multilayer perception with one hidden layer and back propagation learning algorithm."

Subsequently, neural networks have turned out to be a practical way to predict context for many useful use cases. However, the main flaw of this approach is the use of the black box which limits the detection of the exact regularities.

The active LeZi [28], [29] algorithm was proposed as good candidate for context prediction. This algorithm is based on the LZ78 compression data algorithm of Abraham Lempel and Jacob Ziv. It exploits the information of the user's context behavior by using a sliding window to perform a sorting and determine the probabilities for each likely context transition.

Ensemble-learning algorithms: Multiple classifiers can improve the performance of context prediction by exploiting the advantage of individual classifiers in data parts [30]. There are several ways of combining individual classifiers, and the most popular are:

- Voting [30]: Each base classifier predicts a class depending of the number of votes.

- Bagging [30]: Several training subsets Ei are created from the initial training set $\mathrm{E}$ by random re-sampling with replacement. A base classifier is obtained from each training subset. Using vote base classifiers, the final class is selected [30].

However, to our knowledge, the cited works with their techniques have rarely modeled context in an ontological form. In contrast, this model is mainly characterized by its ability to be shared and reused through the inclusion of semantic relationships between the contextual items and the predicted location. Moreover, the number of contexts used to predict the next location was restricted to just two location and time rarely involving other relevant contextual information that would help predict future location more authentically and accurately. Thus, we consider the use of a pattern model on an ontological model with contexts to be more efficient.

\section{PROPOSED FRAMEWORK}

A general overview of the proposed prediction process is shown in Fig. 1. It illustrates the different components of the environment and shows how a user or objects supply events. These components are as follows:

- Environment: It is the milieu where the system exists; it contains the user, objects, places, and sensors.

- Ontology: It is the knowledge base that presents each feature in the environment, context, and patterns of scenarios that have happened previously.

- Location prediction: This module has the interaction context as input [10] from the environment and the predicted location as output. The aim of this module is to use the patterns stored in the ontology to predict the next location. This is done by creating a research pattern which corresponds with the existent pattern in the ontology to find the adequate next location.

- Adequate services: This module's role is to provide adequate services according to location. It has the predicted location as input and the adequate services as output. The module allows interaction with the ontology to select the adequate services depending of the location.

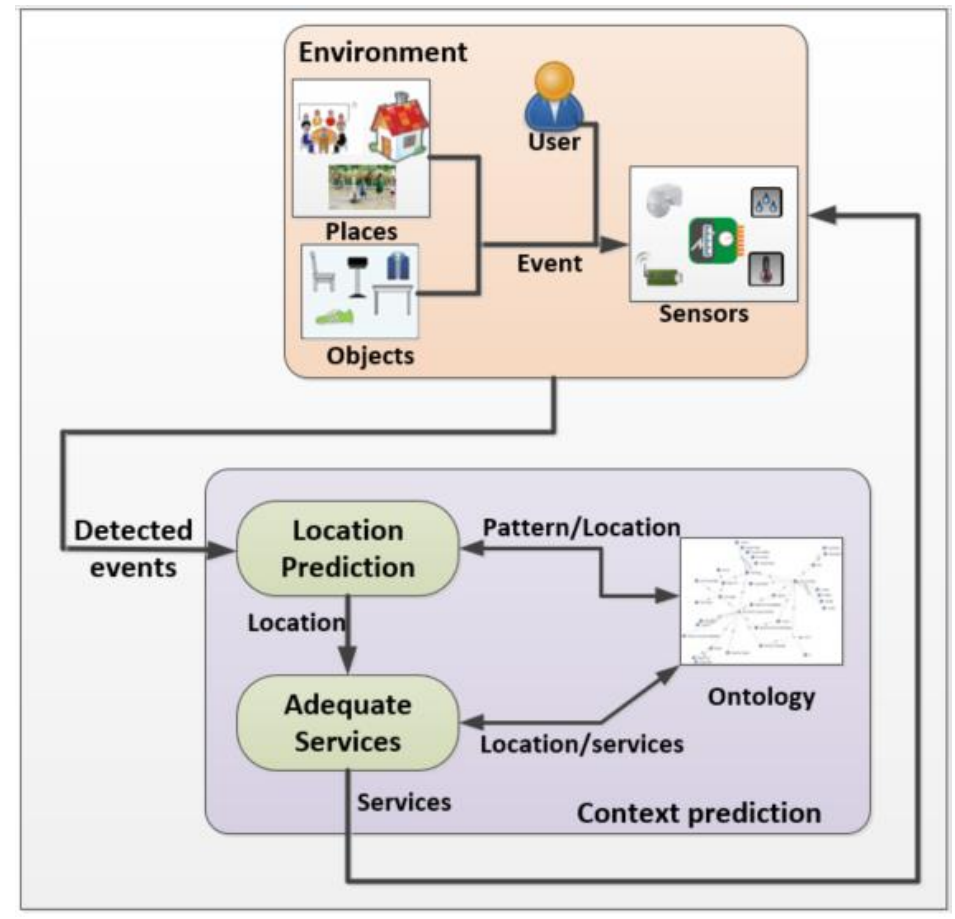

Fig. 1. General View of the Prediction Process. 


\section{APPROACH: MOBILITY PREDICTION: PATTERN TECHNIQUE}

In this section, a detailed approach is presented regarding location prediction. Predicting a future location will allow the provision of adequate services to the user in advance. consequently, the system will be proactive. For example, if the next location is a mall, the system will provide lists of things to buy or will supply lists of shops with sales under way.

\section{A. Pattern}

In this paper, the prediction process is founded on the use of the pattern technique. Usually, these patterns are created by two parts: problem and solution (Fig. 2).

In this paper, these patterns are defined based on users' context history or habits and they are stored in an ontology. Fig. 3 shows a simple model of a pattern.

Therefore, the problem is the command parameters that contain the actual contexts and the user's mobility history. The solution is the predicted location. A real example is shown in Fig. 4.

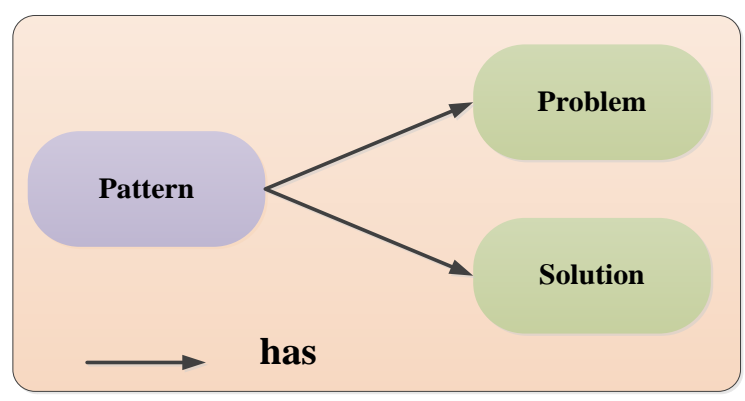

Fig. 2. Pattern Definition.

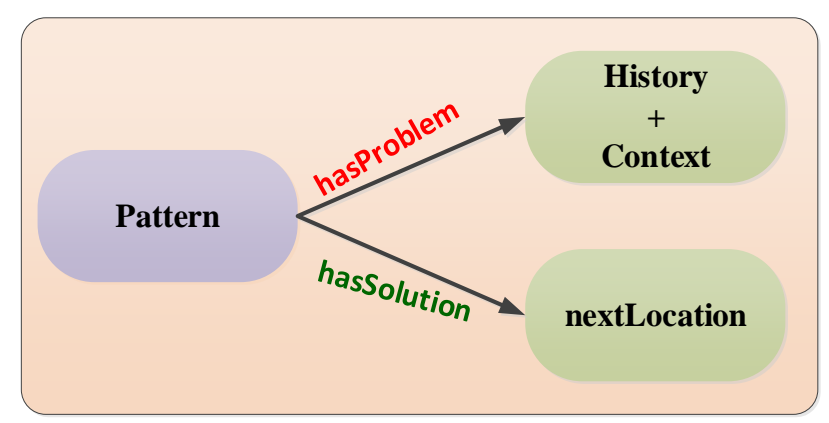

Fig. 3. Example of a Pattern.

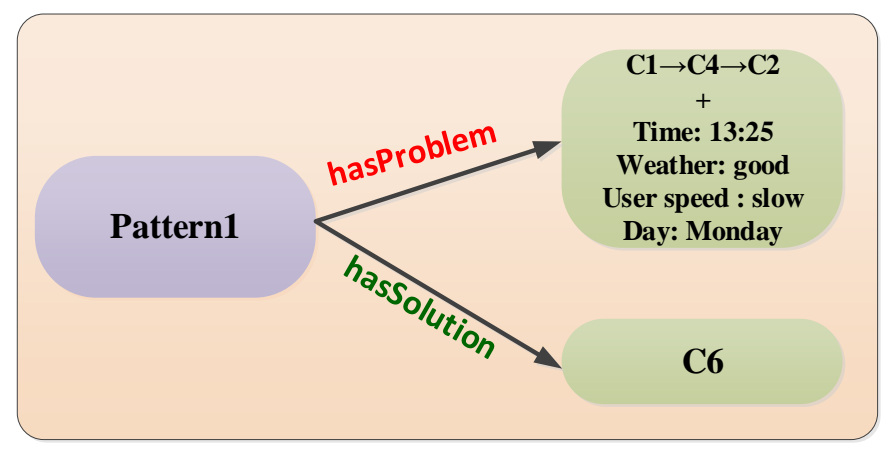

Fig. 4. Real Example of a Pattern.

\section{B. Algorithm}

The detailed algorithm is shown in Fig. 5. As presented, the algorithm has user mobility history, events, and the actual context as input and the next location as output. If an event is detected, then the system creates 'patternSearch', which comprises user mobility history and the actual contexts. This information is collected from the sensors installed in the environment. Then, a connection to the ontology will be established by sending a query containing the 'patternSearch' to get the matching pattern in the knowledge base.

\section{Ontology Creation}

As mentioned before, the role of the ontology is to describe the environment surrounding the user and the prediction system by using a tool called PROTÉGÉ. This tool is based on the ontology web language (OWL). OWL [31], [32] is a language used to define and instantiate Web ontologies. The benefit of using the ontology lies in its ability to be shared and include semantics. Fig. 6 represents the main concepts needed to describe the context and patterns.

The environment is created by the following classes:

- The context class contains the user context, the system context, and the environment context. Consequently, its role is to present the context feature. In this paper, the user context has the subclass user locomotion speed and the environment class has the subclass weather day and time. The system context will be used in a future work.

- The patterns class contains 2,500 subclasses. Every pattern contains two subclasses: problem and solution.

\section{- Algorithm: Pattern Matching}

\section{Input: user history, events, context}

\section{Output: nextLocation}

\section{If event detected then}

Create patternSearch

Connect to the ontology

found $\leftarrow$ False

While (found==False)

$\mid \begin{aligned} & \text { If }(\text { patternSearch }==\text { patternProblem }) \text { then } \\ & \begin{array}{l}\text { nextLocation } \leftarrow \text { PatternSolution } \\ \text { found } \leftarrow \text { True } \\ \text { Else } \\ \text { Go next patternProblem } \\ \text { end }\end{array}\end{aligned}$

end

end

return nextLocation

Fig. 5. Prediction Algorithm. 


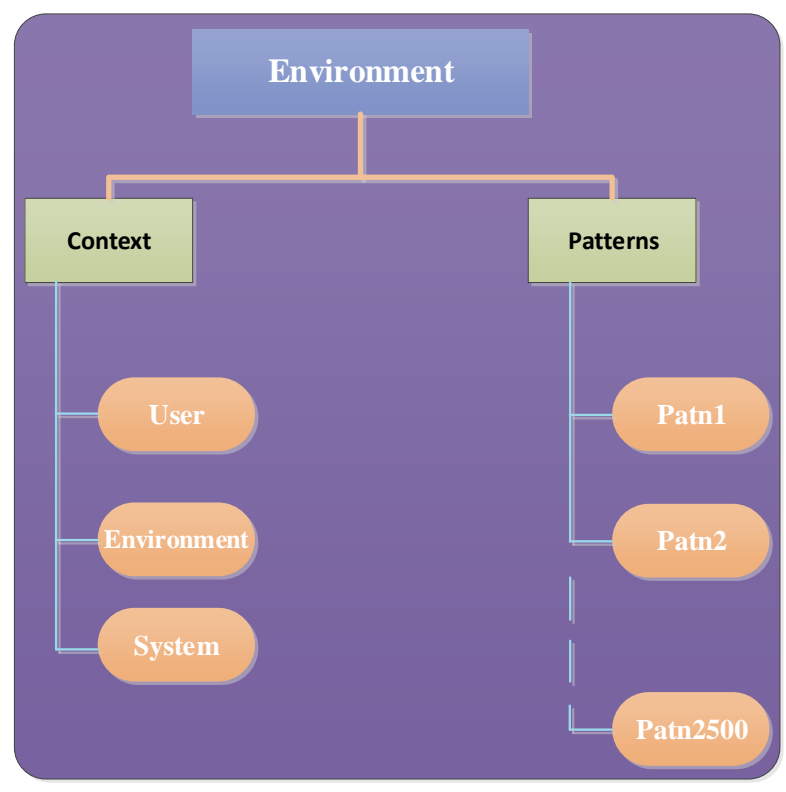

Fig. 6. Ontological Classes.

\section{Use CASE AND Results}

To generate a user's behavior, the MDC (Mobile Data Challenge) database was used, which was created by the Idiap Research Institute from 2009 to 2011 by collecting continuous data from 200 users which described the user's behavior, mobility data, social interactions, and phone usage. The public data set used in this paper contains 38 participants. This data set is divided as follows:
- Speed: 4 ranges; very slow, slow, normal, and fast.

- Time : 6 ranges ; t-0-8, t-8-16, and t-16-24.

- Weather: 3 ranges: bad weather, normal weather, and good weather.

There are many dynamic variables such as time, speed, actual location, weather conditions, days. The way they are integrated can affect decisions. More variables can be added, if it may affect the result.

In this research, $60 \%$ of the data set was used as learning to determine the patterns and $40 \%$ as testing. The patterns are created after the application of the cluster for every day. Then, 2,500 patterns are defined. These patterns are modeled in Fig. 7.

As shown in Fig. 7, for instance, pattern 4 is composed of:

- Pattern_problem_4:

history

location $(\mathrm{C} 2$ P $\rightarrow \mathrm{C} 9$ P $\rightarrow \mathrm{C} 4$ P $\rightarrow \mathrm{C} 6$-P) and contexts of time (12-20), day (Sunday), and weather (good weather).

- Pattern_solution_4: C1_6

The patterns are created according to the cluster of the data set. For instance, Fig. 8 shows a part of the file for Wednesday.

As we can see in Fig. 8, in column $\mathrm{C}$ (the column of the cluster), the user moves from location $\mathrm{C} 2$ to $\mathrm{C} 3$ to $\mathrm{C} 1$ to $\mathrm{C} 4$ to C7 to C6. Therefore, in this case, the pattern is: pattern problem: $\mathrm{C} 2 \rightarrow \mathrm{C} 3 \rightarrow \mathrm{C} 1 \rightarrow \mathrm{C} 4 \rightarrow \mathrm{C} 7$, pattern solution: $\mathrm{C} 6$.

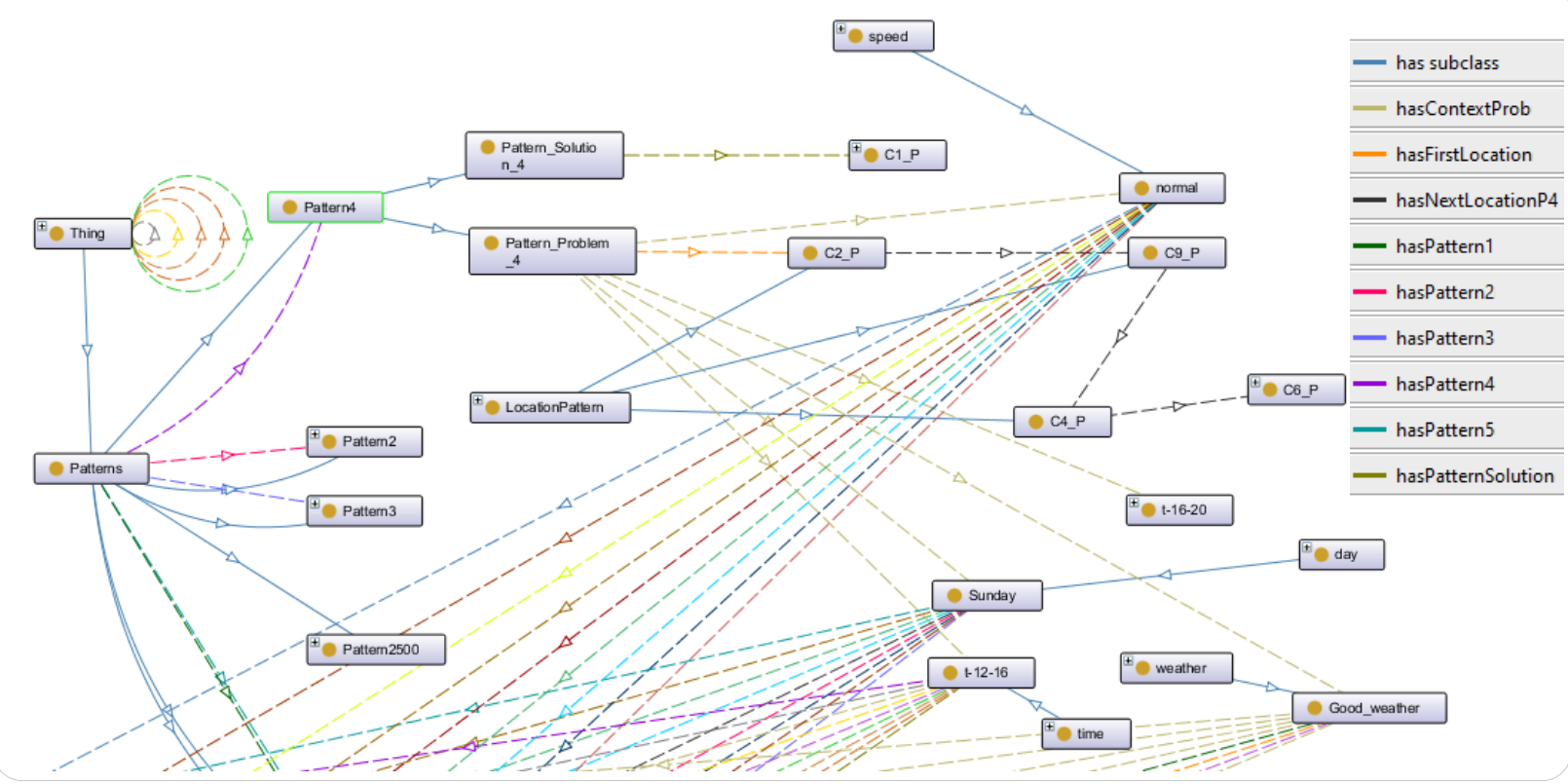

Fig. 7. Ontology. 


\begin{tabular}{|c|c|c|c|c|c|}
\hline A & B & C & D & $E$ & F \\
\hline 6.6213 & 46.515 & 2 & & 5.22 & 6.732 \\
\hline 6.6207 & 46.515 & 2 & & 7523 & 5.976 \\
\hline 6.6203 & 46.515 & 2 & & 55.23 & 10.224 \\
\hline 6.6201 & 46.514 & 2 & & $75: 23$ & 8.964 \\
\hline 6.619 & 46.514 & 2 & & 15.23 & 6.12 \\
\hline 6.6176 & 46.515 & 2 & & 5.25 & 8.208 \\
\hline 6.6232 & 46.516 & 2 & & 6.02 & 2.052 \\
\hline 6.6236 & 46.516 & 2 & & 6.02 & 3.636 \\
\hline 6.6242 & 46.515 & 2 & & 6.03 & 3.852 \\
\hline 6.6268 & 46.515 & 2 & & 6.05 & 6.372 \\
\hline 6.629 & 46.514 & 2 & & 6.07 & 4.392 \\
\hline 6.6292 & 46.514 & 2 & & 6.07 & 4.392 \\
\hline 6.63 & 46.513 & 2 & & 6.08 & 7.2 \\
\hline 6.63 & 46.513 & 2 & & 6.08 & 4.86 \\
\hline 6.6326 & 46.513 & 2 & & 6.10 & 3.06 \\
\hline 6.6332 & 46.513 & 2 & & 6.10 & 3.06 \\
\hline 6.6333 & 46.514 & 2 & & 6.11 & 3.06 \\
\hline 6.6341 & 46.515 & 2 & & $7: 14$ & 6.948 \\
\hline 6.6341 & 46.515 & 2 & & $7: 14$ & 6.948 \\
\hline 6.6302 & 46.516 & 2 & & $7: 17$ & 6.948 \\
\hline 6.6309 & 46.516 & 2 & & $7: 21$ & 4.68 \\
\hline 6.8336 & 46.468 & 3 & & $7: 33$ & $w$ \\
\hline 6.9111 & 46.434 & 1 & & $7: 42$ & $\mathrm{w}$ \\
\hline 6.92 & 46.39 & NOISE & E1- & $7: 45$ & 109.84 \\
\hline 6.92 & 46.39 & NOISE & $1-$ & $7: 46$ & 109.84 \\
\hline 6.9536 & 46.317 & 4 & & 7.50 & 109.84 \\
\hline 6.9638 & 46.316 & 4 & & 7.52 & 1188 \\
\hline 6.9841 & 46.264 & NOISE & SE 4- & $7: 56$ & 1188 \\
\hline 6.999 & 46.237 & MOISE & Nd SE 4- & $7: 58$ & 107.78 \\
\hline 7.0296 & 46. 183 & NOISE & NOSAE 4- & 802 & $w$ \\
\hline 7.0257 & 46.169 & Noise & NOS: 4 - & 802 & $\mathrm{~W}$ \\
\hline 7.0274 & 46.166 & Noise & Noss 4- & 8.02 & 125.46 \\
\hline 7.0286 & 46. 164 & MOISE & Nos: $4-$ & 8.03 & 125.46 \\
\hline 7.031 & 46.158 & NOISE & de 4- & 8.03 & 125.46 \\
\hline 7.06 & 46.1 & 7 & & 2.08 & 5.724 \\
\hline 7.0787 & 46.107 & 6 & & 811 & 8.856 \\
\hline - ..... & $\ldots \ldots$ & & & $\cdot$ & $\ldots$ \\
\hline
\end{tabular}

Fig. 8. Excel File Clustering.

The different algorithms were presented in the related work section. These algorithms were implemented in [33] and they were compare the results obtained by every algorithm using a real data set. The same data set is used, in this paper, for the simulation. Compared to the result obtained in [33] as shown in Table 1, the results obtained are:

The diagram result chart (Fig. 9) sums up the prediction results for each algorithm. In this chart, each line represents the location-prediction accuracy for each algorithm. The horizontal axis represents the days and the vertical axis represents the accuracy.

As shown, the obtained results are unreliable compared to the other algorithms. This is due to the existence of many similar patterns. For instance:

Pattern problem: $\mathrm{C} 1 \rightarrow \mathrm{C} 3 \rightarrow \mathrm{C} 5$, Pattern solution: $\mathrm{C} 7$

Pattern problem: $\mathrm{C} 1 \rightarrow \mathrm{C} 3 \rightarrow \mathrm{C} 5$, Pattern solution: $\mathrm{C} 4$

Pattern problem: $\mathrm{C} 1 \rightarrow \mathrm{C} 3 \rightarrow \mathrm{C} 5$, Pattern solution: $\mathrm{C} 6$

Therefore, for the same pattern problem, there are many solutions. To avoid this issue, all similar patterns are discarded and didn't take them into consideration for the next first results
(Fig. 10, Table 2). Then, we select randomly, in case of similarity (ambiguity), one of the similar patterns (Fig. 11, Table 3).

In conclusion, a remarkable improvement of the result is noted. Therefore, the similar patterns influence the results in an odd way. Therefore, to avoid ambiguity and uncertain decisions, a probabilistic method will be introduced to overcome this issue in future work.

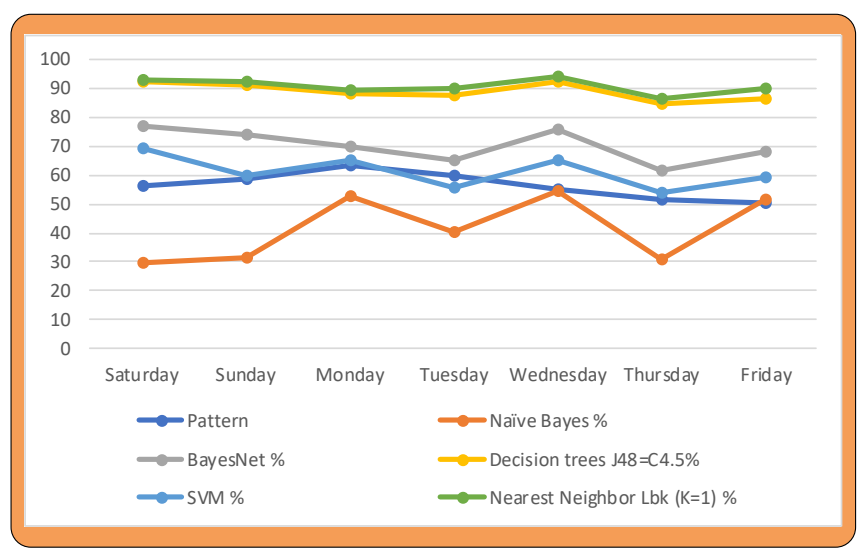

Fig. 9. Diagram result chart

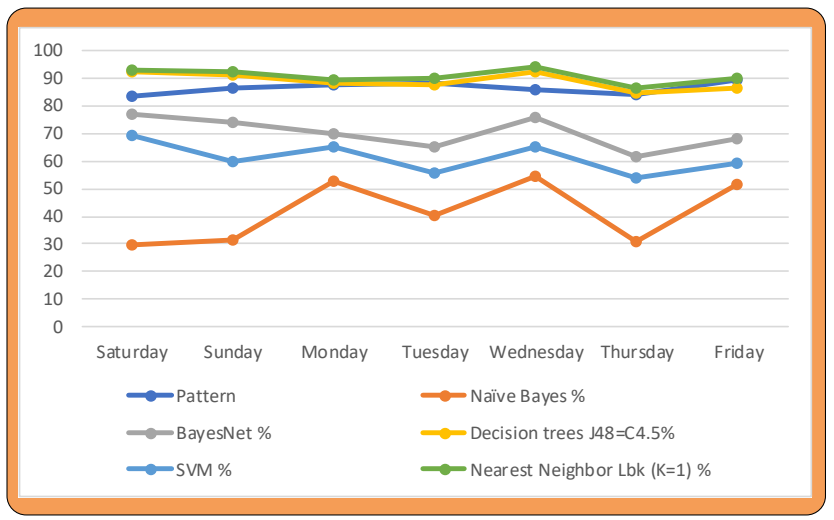

Fig. 10. Diagram result chart without similar patterns

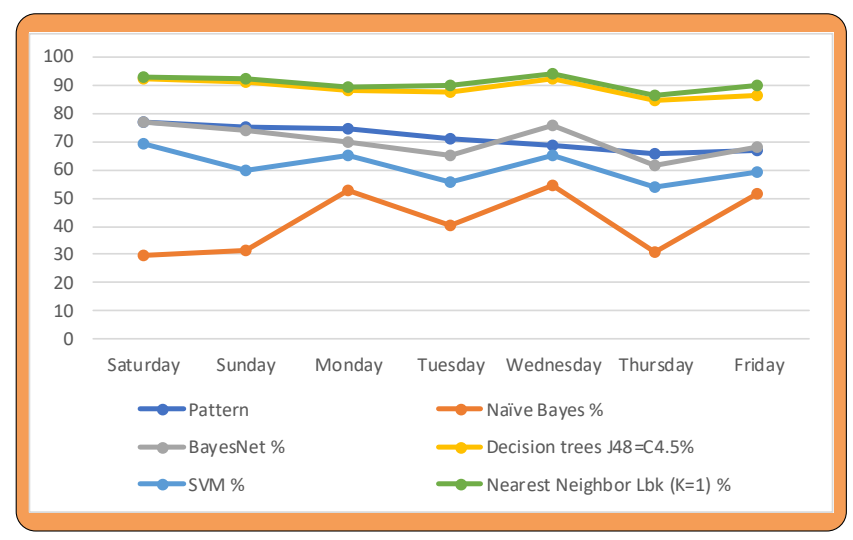

Fig. 11. Diagram result chart with a randomly similar pattern 
TABLE I. MOBILITY PREDICTION ACCURACY [33]

\begin{tabular}{|l|l|l|l|l|l|l|}
\hline Day & Pattern & $\begin{array}{l}\text { Naïve Bayes } \\
\%\end{array}$ & $\begin{array}{l}\text { BayesNet } \\
\%\end{array}$ & $\begin{array}{l}\text { Decision trees } \\
\text { J48=C4.5\% }\end{array}$ & $\begin{array}{l}\text { SVM } \\
\%\end{array}$ \\
\hline Saturday & 56.35 & 29.69 & $\mathbf{7 7 . 3 0}$ & $\mathbf{9 2 . 3 7}$ & $\mathbf{6 9 . 5 4}$ & $\mathbf{9 3 . 2 0}$ \\
\hline Sunday & $\mathbf{5 8 . 8 5}$ & $\mathbf{3 1 . 6 4}$ & $\mathbf{7 4 . 2 5}$ & $\mathbf{9 1 . 2 2}$ & $\mathbf{5 9 . 5 6}$ \\
\hline Monday & $\mathbf{6 3 . 2 1}$ & $\mathbf{5 2 . 9 4}$ & $\mathbf{6 9 . 7 3}$ & $\mathbf{8 8 . 0 6}$ & $\mathbf{6 5 . 4 3}$ & $\mathbf{9 2 . 4 6}$ \\
\hline Tuesday & $\mathbf{6 0 . 0 5}$ & $\mathbf{4 0 . 3 6}$ & $\mathbf{6 5 . 1 8}$ & $\mathbf{8 7 . 5 8}$ & $\mathbf{5 5 . 6 9}$ & $\mathbf{8 9 . 4 7}$ \\
\hline Wednesday & $\mathbf{5 5 . 3 3}$ & $\mathbf{5 4 . 8 0}$ & $\mathbf{7 5 . 6 0}$ & $\mathbf{9 2 . 1 1}$ & $\mathbf{6 5 . 0 4}$ & $\mathbf{9 3 . 9 8}$ \\
\hline Thursday & $\mathbf{5 1 . 4 5}$ & $\mathbf{3 1 . 1 0 0}$ & $\mathbf{6 1 . 4 1}$ & $\mathbf{8 4 . 7 2}$ & $\mathbf{5 4 . 1}$ & $\mathbf{8 6 . 3 9}$ \\
\hline Friday & $\mathbf{5 0 . 6 5}$ & $\mathbf{5 1 . 2 6}$ & $\mathbf{6 7 . 9 6}$ & $\mathbf{8 6 . 7 2}$ & $\mathbf{8 9 . 8 9}$ \\
\hline
\end{tabular}

TABLE II. RESUlts OBTAINED WITHOUT Similar PATtERnS

\begin{tabular}{|l|l|l|l|l|l|l|l|}
\hline Day & Saturday & Sunday & Monday & Tuesday & Wednesday & Thursday \\
\hline Pattern & $\mathbf{8 3 . 5 2}$ & $\mathbf{8 6 . 4 7}$ & $\mathbf{8 7 . 8 9}$ & $\mathbf{8 8 . 0 1}$ & $\mathbf{8 5 . 6 2}$ & $\mathbf{8 3 . 9 4}$ & $\mathbf{8 9 . 6 3}$ \\
\hline
\end{tabular}

TABLE III. RESUlTS OBTAINED WITH SimILAR PATtERNS

\begin{tabular}{|l|l|l|l|l|l|l|l|}
\hline Day & Saturday & Sunday & Monday & Tuesday & Wednesday & Thursday & Friday \\
\hline Pattern & $\mathbf{7 7 . 0 2}$ & $\mathbf{7 5 . 2 1}$ & $\mathbf{7 4 . 5 4}$ & $\mathbf{7 0 . 9 6}$ & $\mathbf{6 8 . 8 4}$ & $\mathbf{6 5 . 7 8}$ \\
\hline
\end{tabular}

\section{CONCLUSION}

This paper proposes a new approach to predict future location of the user. This approach is based on the use of patterns modeled on an ontology with many contextual parameters considered important, such as time, weather, locomotion. The pattern is composed essentially of two parts: problem and solution. The approach is tested using a real data set. The result obtained is considered competitive compared to other algorithms in the case of no similar patterns, but the accuracy of the result is unreliable in the case of similarity. We believe that adding another technique, like the Bayesian network, will improve the results. Therefore, future work will involve the integration of the Bayesian network. This approach can be implemented in smart homes or smart cities to reduce energy consumption.

\section{REFERENCES}

[1] M. Weiser, "The computer for the $21<$ supscrpt $>$ st $</$ supscrpt $>$ century," SIGMOBILE Mob. Comput. Commun. Rev., vol. 3, no. 3, pp. 3-11, 1999.

[2] L. Zhang et al., "It starts with igaze: Visual attention driven networking with smart glasses," in Proceedings of the 20th annual international conference on Mobile computing and networking, 2014, pp. 91-102: ACM.

[3] R. E. Roth, "Interactive maps: What we know and what we need to know," Journal of Spatial Information Science, vol. 2013, no. 6, pp. 59$115,2013$.

[4] W. Barfield, Fundamentals of wearable computers and augmented reality. CRC Press, 2015.

[5] A. Artaud de la Ferrière and N. Vallina-Rodriguez, "The scissors and the magnifying glass: Internet governance in the transitional Tunisian context," The Journal of North African Studies, vol. 19, no. 5, pp. 639$655,2014$.
[6] G. Muhammad, M. Masud, S.U, Amin, R. Alrobaea, and M.F Alhamid, "Automatic Seizure Detection in a Mobile Multimedia Framework". IEEE Access, 6, pp.45372-45383, 2018.

[7] D. Perroud, L. Angelini, O. Abou Khaled, and E. Mugellini, "ContextBased Generation of Multimodal Feedbacks for Natural Interaction in Smart Environments," in AMBIENT 2012, The Second International Conference on Ambient Computing, Applications, Services and Technologies, 2012, pp. 19-25.

[8] H. Sid Ahmed, B. Mohamed Faouzi, and J. Caelen, "Detection and classification of the behavior of people in an intelligent building by camera," International Journal on Smart Sensing \& Intelligent Systems, vol. 6, no. 4, 2013.

[9] C.-J. Chen, J.-A. Chen, and Y.-M. Huang, "INTELLIGENT ENVIRONMENTAL SENSING WITH AN UNMANNED AERIAL SYSTEM IN A WIRELESS SENSOR NETWORK," International Journal on Smart Sensing \& Intelligent Systems, vol. 10, no. 3, 2017.

[10] A. Zaguia, C. Tadj, and A. Ramdane-Cherif, "Context-based method using bayesian network in multimodal fission system," International Journal of Computational Intelligence Systems, vol. 8, no. 6, pp. 10761090, 2015.

[11] G. Abowd, A. Dey, P. Brown, N. Davies, M. Smith, and P. Steggles, "Towards a better understanding of context and context-awareness," in Handheld and ubiquitous computing, 1999, pp. 304-307: Springer.

[12] X. Qin, C.-W. Tan, and T. Clemmensen, "Context-Awareness and Mobile HCI: Implications, Challenges and Opportunities," in HCI in Business, Government and Organizations. Interacting with Information Systems: 4th International Conference, HCIBGO 2017, Held as Part of HCI International 2017, Vancouver, BC, Canada, July 9-14, 2017, Proceedings, Part I, F. F.-H. Nah and C.-H. Tan, Eds. Cham: Springer International Publishing, 2017, pp. 112-127.

[13] C. Perera, A. Zaslavsky, P. Christen, and D. Georgakopoulos, "Context aware computing for the internet of things: A survey," IEEE Communications Surveys \& Tutorials, vol. 16, no. 1, pp. 414-454, 2014.

[14] B. Yuan and J. Herbert, "Context-aware hybrid reasoning framework for pervasive healthcare," Personal and ubiquitous computing, vol. 18, no. 4, pp. 865-881, 2014. 
[15] T. M. T. Do and D. Gatica-Perez, "Where and what: Using smartphones to predict next locations and applications in daily life," Pervasive and Mobile Computing, vol. 12, pp. 79-91, 2014.

[16] D. Zhang, D. Zhang, H. Xiong, L. T. Yang, and V. Gauthier, "NextCell: predicting location using social interplay from cell phone traces," IEEE Transactions on Computers, vol. 64, no. 2, pp. 452-463, 2015.

[17] Y. Wang et al., "Regularity and conformity: Location prediction using heterogeneous mobility data," in Proceedings of the 21th ACM SIGKDD International Conference on Knowledge Discovery and Data Mining, 2015, pp. 1275-1284: ACM.

[18] C. A. Williams, A. Mohammadian, P. C. Nelson, and S. T. Doherty, "Mining sequential association rules for traveler context prediction," presented at the Proceedings of the 5th Annual International Conference on Mobile and Ubiquitous Systems: Computing, Networking, and Services, Dublin, Ireland, 2008.

[19] J. Hong, E.-H. Suh, J. Kim, and S. Kim, "Context-aware system for proactive personalized service based on context history," Expert Systems with Applications, vol. 36, no. 4, pp. 7448-7457, 2009.

[20] A. Boytsov, "Context reasoning, context prediction and proactive adaptation in pervasive computing systems," Luleå tekniska universitet, 2011.

[21] S. Chang, D.-z. WU, X.-z. XIE, and W. Qi, "Temporal Markov Chain Location Prediction," DEStech Transactions on Materials Science and Engineering, no. ammme, 2016.

[22] A.Alsufyani, O. Hajilou, A. Zoumpoulaki, M. Filetti, H. Alsufyani, C.J. Solomon, S.J Gibson, R. Alroobaea and H. Bowman, "Breakthrough percepts of famous faces". Psychophysiology, 56(1), p.e13279. 2019.

[23] T. Alotaibi, A. Nazir, R. Alroobaea, M. Alotibi, F. Alsubeai, A. Alghamdi, T. Alsulimani, " Saudi Arabia Stock Market Prediction Using Neural Network". International Journal on Computer Science and Engineering (IJCSE), Vol. 10 No.2 .2018.

[24] T. Mikluščák and M. Gregor, "Person movement prediction using artificial neural networks with dynamic training on a fixed-size training data set," Applied computer science: management of production processes, vol. 7, no. 2, pp. 43-56, 2011.

[25] M. C. Mozer, "The neural network house: An environment hat adapts to its inhabitants," in Proc. AAAI Spring Symp. Intelligent Environments, 1998, vol. 58.

[26] L. Vintan, A. Gellert, J. Petzold, and T. Ungerer, "Person movement prediction using neural networks," 2006.

[27] T. Lin, C. Wang, and P.-C. Lin, "A neural-network-based context-aware handoff algorithm for multimedia computing," ACM Transactions on Multimedia Computing, Communications, and Applications (TOMM), vol. 4 , no. 3, p. $17,2008$.

[28] M.-J. Tsai et al., "Context-aware activity prediction using human behavior pattern in real smart home environments," in Automation Science and Engineering (CASE), 2016 IEEE International Conference on, 2016, pp. 168-173: IEEE.

[29] K. Gopalratnam and D. J. Cook, "Online sequential prediction via incremental parsing: The active lezi algorithm," IEEE Intelligent Systems, vol. 22, no. 1, 2007.

[30] T. Anagnostopoulos, C. Anagnostopoulos, S. Hadjiefthymiades, M. Kyriakakos, and A. Kalousis, "Predicting the location of mobile users: a machine learning approach," in Proceedings of the 2009 international conference on Pervasive services, 2009, pp. 65-72: ACM.

[31] G. Antoniou and F. Harmelen, "Web Ontology Language: OWL," in Handbook on Ontologies, S. Staab and R. Studer, Eds. (International Handbooks on Information Systems: Springer Berlin Heidelberg, 2009, pp. 91-110.

[32] Z. Huiqun, Z. Shikan, and Z. Junbao, "Research of Using Protege to Build Ontology," in Computer and Information Science (ICIS), 2012 IEEE/ACIS 11th International Conference on, 2012, pp. 697-700.

[33] D. Guessoum, D. Guessoum, M. Miraoui, M. Miraoui, C. Tadj, and C. Tadj, "Contextual location prediction using spatio-temporal clustering," International Journal of Pervasive Computing and Communications, vol. 12, no. 3, pp. 290-309, 2016. 